\title{
Techniques for Noise and Nonlinear Impairments Compensation in CO-OFDM Transmission
}

\author{
Son Thai Le* and Sergei K. Turitsyn \\ Aston Institute of Photonic Technologies (AIPT), Aston University, UK \\ e-mail: let1@aston.ac.uk
}

\begin{abstract}
In this paper, we discuss recent advances in digital signal processing techniques for compensation of the laser phase noise and fiber nonlinearity impairments in coherent optical orthogonal frequency division multiplexing (CO-OFDM) transmission. For laser phase noise compensation, we focus on quasi-pilot-aided (QPA) and decision-directed-free blind (DDF-blind) phase noise compensation techniques. For fiber nonlinearity compensation, we discuss in details the principle and performance of the phase-conjugated pilots (PCP) scheme. Keywords: Optical communications, coherent optical orthogonal frequency division multiplexing, laser phase noise compensation, fiber nonlinearity compensation
\end{abstract}

\section{INTRODUCTION}

Coherent optical orthogonal frequency division multiplexing (CO-OFDM) has been considered as a promising candidate for long-haul optical communication systems because of the high spectral efficiency offered and excellent tolerance towards linear fiber impairments, such as chromatic dispersion and polarization mode dispersion [1,2]. However, in comparison to single carrier transmission schemes, OFDM also provides a lot of challenges. Firstly, resulted from the relatively long symbol duration, OFDM is more sensitive to laser phase noise, which introduces both common phase error (CPE) and intercarrier interference (ICI), which seriously degrade the system performance [3]. Secondly, because of the narrow and equal spacing of subcarrier, OFDM suffers from a number of nonlinear effects, especially the four-wave-mixing (FWM) [4, 5]. As a result, the design, development, and operation of CO-OFDM systems all require efficient methods for compensating laser phase noise and fiber nonlinearity impairments. In this paper, we discuss in details effective digital signal processing (DSP) techniques for the compensation of laser phase noise and fiber nonlinearity impairments in CO-OFDM transmission, including quasi-pilot-aided (QPA), decision-directed-free blind (DDF-blind) and phase-conjugated pilots (PCP) techniques.

\section{PHASE NOISE COMPENSATION IN CO-OFDM TRANSMISSION}

2.1 Quasi-pilot-aided phase noise estimation.

Existing CO-OFDM phase noise compensation (PNC) techniques may be divided into three groups, data aided (DA), pilot aided (PA) and RF-pilot [6]. Among these methods PA is the most widely used method due to its inherently low complexity and high precision [7]. However, the ultimate shortcoming of PA PNC is the required additional overhead. Recently, a novel PNC technique called QPA has been proposed in [8, 9], which allows the overhead to be reduced by a factor of 2 while offering a similar performance in comparison to PA method. The key idea behind QPA methods is setting the pilot subcarriers (PS) in correlation with other data-carrying subcarriers. In the other word, PSs in QPA scheme are data dependent, unlike the conventional PA scheme where PSs are predetermined. The correlation among PSs and data-carrying subcarriers can be chosen in different ways, for example "phase conjugate" and "phase parity". In the first scheme (phase conjugate or QPA1), each pilot subcarrier is the complex conjugate of the data carrying subcarrier equally spaced from the central reference. At the receiver each pilot subcarrier is coherently combined with its data carrying counterpart, eliminating the data modulation and enhancing the signal to noise ratio [8,9]. In the second scheme (phase parity pilot or QPA-2), the phases of pilot subcarriers are chosen such that their mean phase angle is opposite that of all the remaining data subcarriers. In this case at the receiver, the CPE is estimated by summing the phases of all pilots and associated data subcarriers $[8,9]$.

The two QPA PNC schemes are experimentally compared with RF and PA methods as a function of the OSNR, as shown in Fig. 1 (a) for a combined laser linewidth of $200 \mathrm{kHz}$. In Fig. 1(a), both QPA schemes outperform the PA PNC scheme and in particular 2 pilot QPA schemes offer similar performance to 4-pilot PA schemes indicating that the overhead can be effectively reduced by a factor of 2. Similarly, 2-pilot QPA outperforms RFpilot tone. On the other hand, the RF-pilot tone method is strongly affected by the size of the frequency guard band surrounding the DC subcarrier; consequently with a small overhead (1\% in this experiment) the QPA method outperforms the RF method. In particular, 2-pilot QPA requires almost equivalent overhead to that of the RF-pilot tone in the system under investigation. The impact of overhead on CPE estimation performance is illustrated in Fig. 1(b), showing BER at an experimentally measured OSNR of $\sim 26 \mathrm{~dB}$. With the conventional PA method, 10 pilots are required for negligible penalty. On the other hand, for both QPA schemes the required number of PSs is less than 6, a reduction of almost a factor of 2 . 

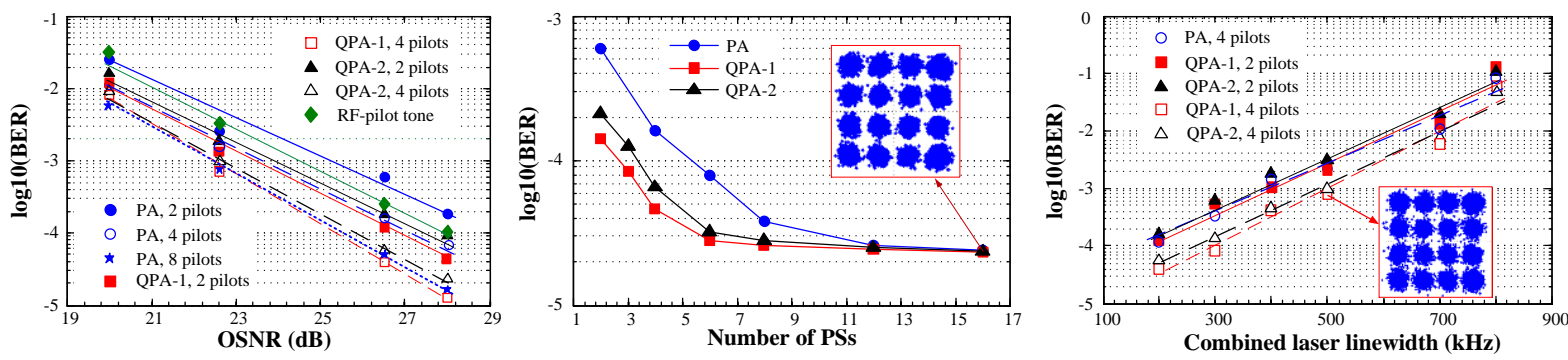

Fig. 1: Experimental results for (a) BER versus OSNR with $200 \mathrm{kHz}$ combined linewidth, (b) BER versus number of PSs at 26dB ONSR and (c) BER versus combined laser linewidth also at 26dB OSNR.

\subsection{Decision-Directed-Free Blind Phase noise estimation}

Blind PNC based on a decision-directed (DD) for CO-OFDM was considered in [10]. However, this technique suffers significantly from error propagation, and thus, it cannot be applied in the presence of a large laser phase noise. In [11] a hardware efficient DDF-blind phase noise estimation method, which is unaffected by error propagation, was proposed. In this method [11] a novel cost function, which is the mean value of the squared product of the projections of real and imaginary parts after rotation by a phase angle $\phi$, was introduced as:

$$
\mathrm{F}_{m}(\phi)=\left\langle\operatorname{Re}\left(R_{m, k} \cdot e^{-j \phi}\right)^{2} \cdot \operatorname{Im}\left(R_{m, k} \cdot e^{-j \phi}\right)^{2}\right\rangle_{k},
$$

where $<\cdot>$ stands for the averaging operation over all of the subcarriers in the $m^{\text {th }}$ symbol.

For squared QAM modulation formats with identical probabilities of constellation points, this cost function reaches its maximum value at $\phi=\Phi_{\mathrm{m}}$, where $\Phi_{\mathrm{m}}$ is the CPE of the $m^{\text {th }}$ symbol due to the laser phase noise. This phenomenon can be explained by the fact that ideal squared QAMs provide a "balance" between the real and imaginary parts of constellation points, thus, maximizing the mean value of the squared product of the projections of real and imaginary parts. The cost function (1) can be approximated as [11]:

$$
\mathrm{F}_{m}(\phi)=A \cos \left(4 \phi-4 \Phi_{m}\right)+B
$$

where $A, B, \Phi_{m}$ are three variables to be determined. Knowing the form of the cost function, the CPE $\left(\Phi_{m}\right)$ can be easily defined using just three test phases, for example, $0, \pi / 4$ and $\pi / 8$ as follows:

$$
\begin{array}{r}
B=\left(\mathrm{F}_{m}(0)+\mathrm{F}_{m}(\pi / 4)\right) / 2, A=\sqrt{\left(\mathrm{F}_{m}(0)-B\right)^{2}+\left(\mathrm{F}_{m}(\pi / 8)-B\right)^{2}} \\
\Phi_{m}=0.25 \cdot \operatorname{sgn}\left(B-\mathrm{F}_{m}(\pi / 8)\right) \cdot \operatorname{acos}\left(\left(\mathrm{F}_{m}(0)-B\right) / A\right),
\end{array}
$$

where $\operatorname{sgn}()$ is the sign function.
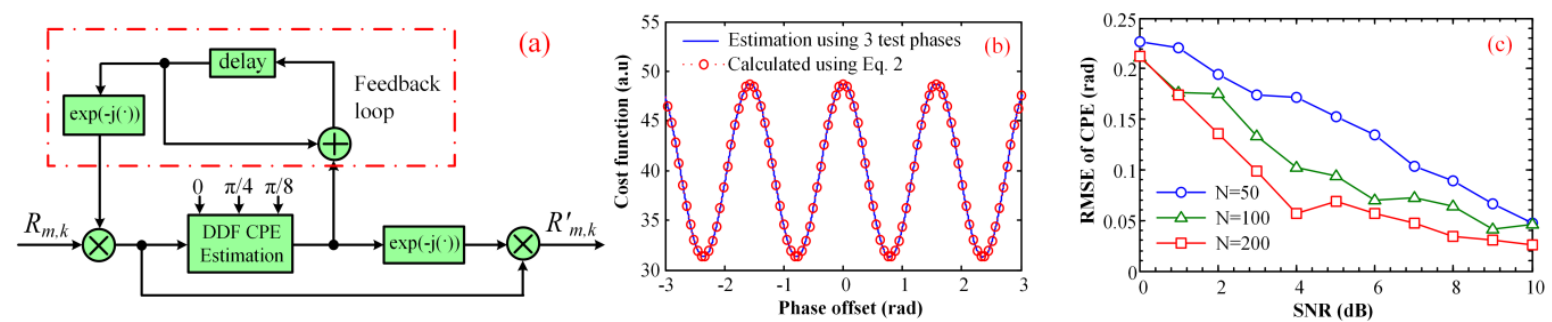

Fig. 2. (a) - Schematic diagram of DDF blind PNC technique for CO-OFDM, (b) - The cost function of 16QAM at a SNR of 4dB, (c) - CPE error as a function of SNR for 16QAM for different values of the number of calculated samples $(\mathrm{N})$

The calculated (using (1)) and estimated (using 3 test phases) cost functions for 16-QAM with a SNR of $4 \mathrm{~dB}$ and $N=200$ are compared in Fig. 2(b). The root-mean-square error (RMSE) of the CPE calculated by (4) is plotted in Fig. 1(c) as a function of the SNR for 16QAM, showing that a small RMSE of 0.1 rad can be achieved for $N=50$ at $\mathrm{SNRs}>7 \mathrm{~dB}$. This result clearly indicates the high tolerance of the proposed CPE estimation method to Gaussian noise.

The performance of DDF blind PNC scheme is compared with blind DD, blind phase search (BPS) [12] and PA methods for single channel 16QAM transmission in Fig. 3(a) for a combined laser linewidth of $200 \mathrm{kHz}$. DDF blind is only matched in performance by highly complex BPS with 16 test phases. A similar comparison result is observed in the Fig. 3(b), which shows the BER as a function of the launch power for the center channel in the WDM QPSK CO-OFDM transmission over $2400 \mathrm{~km}$. The obtained result clearly indicates that DDF blind PNC is also effective in compensating the CPE due to cross-phase modulation in WDM transmissions. In comparison with PA methods, DDF blind PNC offers better performance while avoiding the SE reduction, due to the pilot subcarriers, which is about $7.6 \%$ in this case. This highlights the great benefit of the DDF blind PNC technique. 
Fig. 3(c) shows that DDF blind PNC technique can be applied effectively (without differential coding) with a combined laser linewidth up to $700 \mathrm{kHz}$.
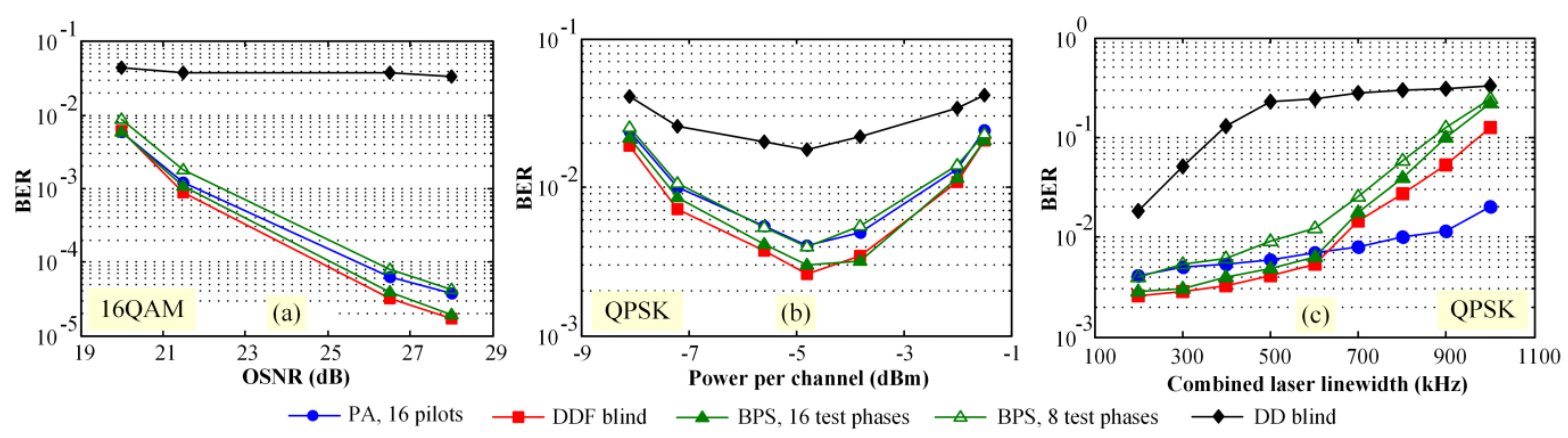

Fig. 3. Experimental results for (a) BER versus OSNR in the back-to-back case, single channel with 16QAM, the combined laser linewidth is $200 \mathrm{kHz}$; (b) BER versus the power for the center channel in QPSK WDM transmission, the distance is 2400km, the combined laser linewidth is $200 \mathrm{kHz}$; (c) BER versus the combined laser linewidth for the center channel in QPSK WDM transmission, the distance is $2400 \mathrm{~km}$, the power is $-4.8 \mathrm{dBm}$.

\section{NONLINEARITY COMPENSATION USING PHASE CONJUGATED PILOTS}

In CO-OFDM transmissions, due to the narrow frequency spacing (tens of $\mathrm{MHz}$ ) the nonlinear phase-shifts of neighbouring subcarriers are essentially correlated, especially when $50 \%$ pre-electrical dispersion compensation (pre-EDC) is applied [13, 14]. Based on this phenomenon, an effective nonlinearity compensation technique for CO-OFDM was proposed in [13] by transmitting a portion of OFDM subcarriers with its phase-conjugates pilots (PCPs, Fig. 4(a)). At the receiver, PCPs are used to estimate the nonlinear distortions in the respective subcarriers (though coherent superposition of PCP pairs, Fig. 4(b)) and other subcarriers, which are not accompanied by PCPs. As a result, effective nonlinearity compensation can be achieved in a flexible way where the overhead can be adjusted according to the required performance gain. This is an advantage of PCP over phase-conjugated twin wave (PC-TW) technique, which always scarifies 50\% of the transmission capacity [15].
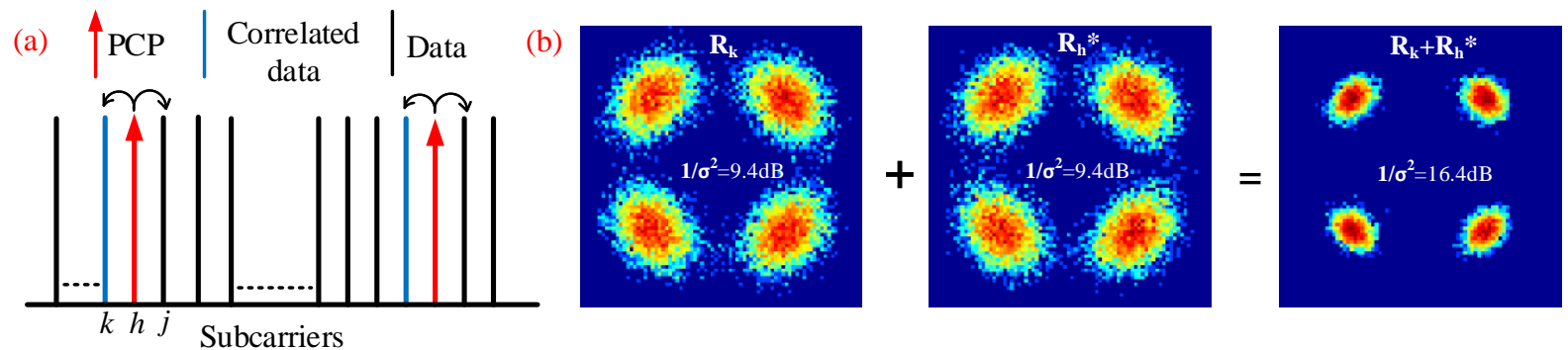

Fig. 4(a) - Inserting phase-conjugated pilots for fibre nonlinearity compensation. (b) - Nonlinear noise cancellation based on the coherent superposition of PCP pairs of $112 \mathrm{~Gb} / \mathrm{s}$ PDM CO-OFDM (20 MHz subcarrier spacing) system over 1200km, launch power is 5dBm, ASE noise is not considered and $50 \%$ pre-EDC is adopted.

The effectiveness of the nonlinear noise cancellation scheme based on the coherent superposition of the PCP pairs is shown in Fig. 4(b), where a dramatic reduction $(\sim 7 \mathrm{~dB})$ of the nonlinear signal distortion variance $\left(\sigma^{2}\right)$ was observed. This clearly indicates that the nonlinear distortion on a data carrying subcarrier and those of its PC are highly anticorrelated, especially if the frequency spacing is small.

The experimental results on the performance of PCPs method in QPSK WDM CO-OFDM transmission is presented in Fig. 5. It can be seen in the Fig. 5 (a) that by transmitting 50\% of OFDM subcarriers as PCPs a dramatic $(\sim 4 \mathrm{~dB})$ improvement in the system's Q-factor was achieved (after $3200 \mathrm{~km}$ of the transmission distance), which is comparable with results achieved with the conventional PC-TW technique [15]. The overhead in PCP scheme can be reduced by using a smaller number of PCPs. Specifically, one PCP can be used for 2, 3, 4 or more data subcarriers at the cost of 33\%, $25 \%, 20 \%$ or smaller overhead respectively. When the PCP overhead was reduced to $25 \%$, a performance improvement of around $1.5 \mathrm{~dB}$ was still achieved (Fig. 5(a)). This result confirms the possibility of using one PCP to compensate the nonlinear distortions of several data subcarriers. Figure 5(b) plots the Q-factor improvement as a function of the additional overhead due to the PCP, showing the trade-off between PCP overhead and performance gain. Since when PCPs were not transmitted, $8 \%$ of OFDM subcarriers were allocated for phase noise estimation, the additional overhead for nonlinear compensations were $12 \%, 17 \%, 25 \%$ and $42 \%$ and the performance gains were $0.9,1.2,1.5$ and $4 \mathrm{~dB}$ respectively. This result clearly shows the flexibility of the proposed PCP fibre nonlinearity compensation technique, allowing the number of PCP to be chosen to meet the performance requirement. 

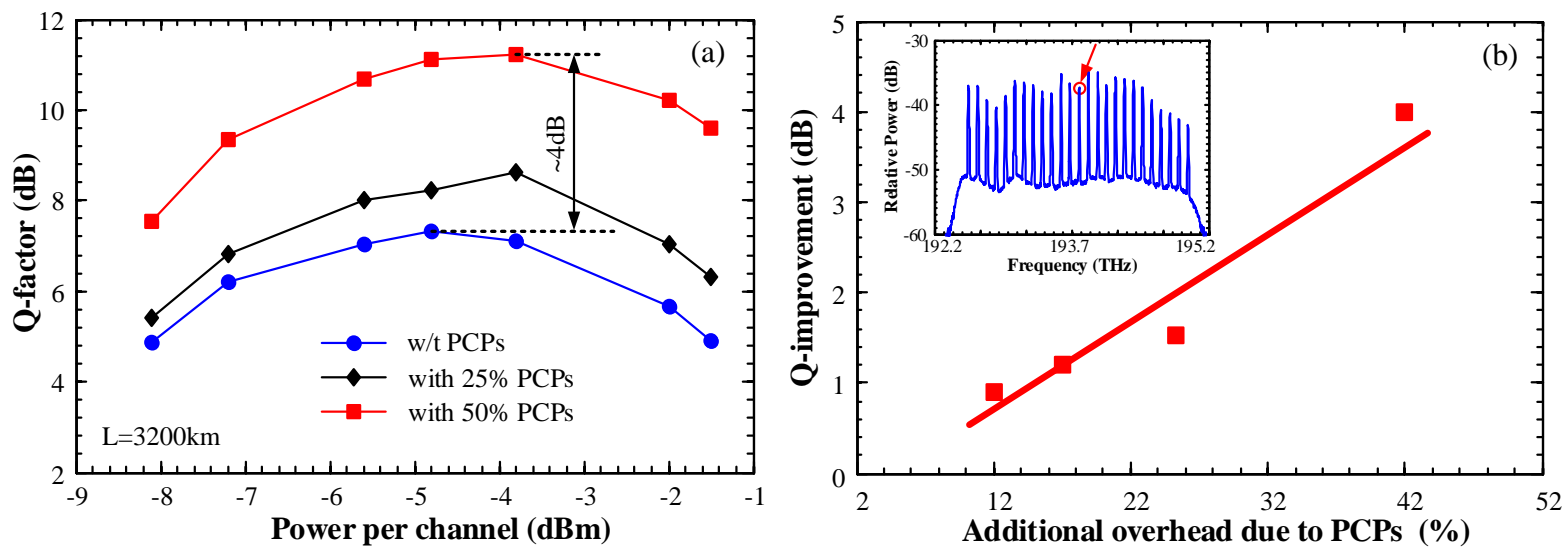

Fig. 5(a) - Experimental comparison of Q-factor of the center channel in a QPSK WDM CO-OFDM system with and without PCPs for fibre nonlinearity compensation as a function of the launch power, (b) - Performance gain as a function of the additional overhead due to PCPs for the center channel. Without PCPs, an overhead of $\sim 8 \%$ was required for CPE compensation, the transmission distance was $3200 \mathrm{~km}$.

\section{CONCLUSIONS}

Laser phase noise and fiber nonlinearity impairments in CO-OFDM transmissions can be effectively mitigated using DSP techniques. In particular, setting pilot subcarriers in correlation with other data-carrying subcarriers is an effective strategy to reduce the overhead by a factor of 2 (with phase conjugate and phase parity techniques). The overhead can also be completely avoided in DDF-blind PNC scheme. In addition, by transmitting a portion of subcarriers as PCPs, the fiber nonlinearity impairments can also be compensated in a flexible way, where the overhead can be adjusted according to the performance gain requirements.

\section{ACKNOWLEDGEMENTS}

This work was partially supported by the UK EPSRC programme Grants UNLOC and PEACE (EP/J017582/1 and EP/L000091/1) and the European Commission's 7th Framework Programme FP/2007-2013 grants 258033 (MODE-GAP) and 318415 (FOX-C), and the support of Sterlite Technologies and Finisar is gratefully acknowledged

\section{REFERENCES}

[1] E. Giacoumidis, M. A. Jarajreh, S. Sygletos, S. T. Le, F. Farjady, A. Tsokanos, et al., "Dual-polarization multi-band optical OFDM transmission and transceiver limitations for up to $500 \mathrm{~Gb} / \mathrm{s}$ uncompensated long-haul links," Optics Express, vol. 22, pp. 1097510986, 2014/05/05 2014.

[2] F. Buchali, R. Dischler, and X. Liu, "Optical OFDM: A promising high-speed optical transport technology," Bell Labs Technical Journal, vol. 14, pp. 125-146, 2009.

[3] S. Wu and Y. Bar-Ness, "OFDM systems in the presence of phase noise: consequences and solutions," Communications, IEEE Transactions on, vol. 52, pp. 1988-1996, 2004.

[4] S. T. Le, K. Blow, and S. Turitsyn, "Power pre-emphasis for suppression of FWM in coherent optical OFDM transmission," Optics Express, vol. 22, pp. 7238-7248, 2014/03/24 2014.

[5] R. Essiambre, G. Kramer, P. J. Winzer, G. J. Foschini, and B. Goebel, "Capacity Limits of Optical Fiber Networks," Lightwave Technology, Journal of, vol. 28, pp. 662-701, 2010.

[6] S. L. Jansen, I. Morita, T. C. W. Schenk, and H. Tanaka, "121.9-Gb/s PDM-OFDM Transmission With 2-b/s/Hz Spectral Efficiency Over 1000 km of SSMF," Lightwave Technology, Journal of, vol. 27, pp. 177-188, 2009.

[7] Y. Xingwen, W. Shieh, and T. Yan, "Phase Estimation for Coherent Optical OFDM," Photonics Technology Letters, IEEE, vol. 19, pp. 919-921, 2007.

[8] S. T. Le, T. Kanesan, M. McCarthy, E. Giacoumidis, I. Phillips, M. F. Stephens, et al., "Experimental Demonstration of Datadependent Pilot-aided Phase Noise Estimation for CO-OFDM," in Optical Fiber Communication Conference, San Francisco, California, 2014, p. Tu3G.4.

[9] L. Son Thai, T. Kanesan, E. Giacoumidis, N. J. Doran, and A. D. Ellis, "Quasi-Pilot Aided Phase Noise Estimation for Coherent Optical OFDM Systems," Photonics Technology Letters, IEEE, vol. 26, pp. 504-507, 2014.

[10] M. E. Mousa-Pasandi and D. V. Plant, "Zero-overhead phase noise compensation via decision-directed phase equalizer for coherent optical OFDM," Optics Express, vol. 18, pp. 20651-20660, 2010/09/27 2010.

[11] S. T. Le, M. E. McCarthy, N. M. Suibhne, P. A. Haigh, E. Giacoumidis, N. J. Doran, et al., "Decision-Directed-Free Blind Phase Noise Estimation for CO-OFDM," presented at the OFC, paper. W1E.5, Los Angeles, 2015.

[12] T. Pfau, S. Hoffmann, and R. Noe, "Hardware-Efficient Coherent Digital Receiver Concept With Feedforward Carrier Recovery for m-QAM Constellations," Lightwave Technology, Journal of, vol. 27, pp. 989-999, 2009.

[13] S. T. Le, M. E. McCarthy, N. M. Suibhne, A. D. Ellis, and S. K. Turitsyn, "Phase-conjugated Pilots for Fibre Nonlinearity Compensation in CO-OFDM Transmission," presented at the ECOC, Cannes, France, paper We.2.3.1, 2014.

[14] S. T. Le, E. Giacoumidis, N. Doran, A. D. Ellis, and S. K. Turitsyn, "Phase-conjugated Subcarrier Coding for Fibre Nonlinearity Mitigation in CO-OFDM Transmission," presented at the ECOC, Cannes, France, paper We.3.3.2, 2014.

[15] X. Liu, R. A. Chraplyvy, P. J. Winzer, W. R. Tkach, and S. Chandrasekhar, "Phase-conjugated twin waves for communication beyond the Kerr nonlinearity limit," Nat Photon, vol. 7, pp. 560-568, 2013. 